Canadian

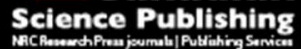

Canadian Journal of Physiology and Pharmacology Revue canadienne de physiologie et pharmacologie

\title{
The cardioprotective effect of naringenin against ischemia/ reperfusion injury through activation of ATP-sensitive potassium channel in rat
}

\begin{tabular}{|r|l|}
\hline Journal: & Canadian Journal of Physiology and Pharmacology \\
\hline Manuscript ID & cjpp-2016-0008.R1 \\
\hline Danuscript Type: & Article \\
\hline Complete List of Authors: & $\begin{array}{l}\text { Meng, Li-Min; General Hospital of FengFeng Branch in Center Hebei Energy } \\
\text { Sources Group } \\
\text { Ma, Hui-Ji; Hebei Medical University, Department of physiology } \\
\text { Guo, Hui; the Fourth Hospital of Hebei Medical University, Department of } \\
\text { gynaecology and obstetrics } \\
\text { Kong, Qian-Qian; Shandong Zaozhuang Vocational College of Technology } \\
\text { Zhang, Yi; Hebei Medical University, Department of Physiology }\end{array}$ \\
\hline Keyword: & $\begin{array}{l}\text { Naringenin, Cardiac protection, Ischemia/reperfusion, ATP-sensitive } \\
\text { potassium channels, Antioxidation }\end{array}$ \\
\hline &
\end{tabular}


Manuscript ID cjpp-2016-0008

\title{
The cardioprotective effect of naringenin against ischemia/
}

\section{reperfusion injury through activation of ATP-sensitive potassium}

\section{channel in rat}

\author{
Li-Min Meng ${ }^{1,2}$, Hui-Jie Ma ${ }^{1,4}$, Hui Guo ${ }^{3}$, Qian-Qian Kong ${ }^{1,5}$, Yi Zhang $^{1,4 *}$ \\ ${ }^{1}$ Department of Physiology, Hebei Medical University, Shijiazhuang 050017, PR
} China;

${ }^{2}$ Second Department of Cardiology, General Hospital of FengFeng Branch in Center Hebei Energy Sources Group, Handan 056200, PR China;

${ }^{3}$ Department of gynaecology and obstetrics, the Fourth Hospital of Hebei Medical University, Shijiazhuang 050000, PR China;

4 Hebei Collaborative Innovation Center for Cardio-cerebrovascular Disease, Shijiazhuang, Hebei 050000, PR China;

${ }^{5}$ Shandong Zaozhuang Vocational College of Technology, Tengzhou, Shandong 277500, PR China

Running title: cardioprotective effect of naringenin

\section{* Corresponding author:}

Yi Zhang, Ph.D, Department of Physiology, Hebei Medical University, Zhong Shan Dong Rd., Shijiazhuang 050017, China. E-mail:yzhang@hebmu.edu.cn, Tel: +86 311 8626 5663, fax: +8631186266811. 


\begin{abstract}
Naringenin (Nari) has antioxidative and anti-atherosclerosis effects, and activation of ATP-sensitive potassium channel $\left(\mathrm{K}_{\mathrm{ATP}}\right)$ can offer a cardiac protection. We hypothesized that Nari protects heart against ischemia/ reperfusion (I/R) injury through activation of $\mathrm{K}_{\mathrm{ATP}}$. Isolated heart from adult male Sprague-Dawley rats experienced a 30-min global ischemia followed by 60 -min reperfusion (120 min for the infarct size determination). The hearts were treated with Nari (NARI), Nari plus glibenclamide (GLI), a non-specific ATP-sensitive potassium channel blocker (NARI+GLI) and Nari plus 5-hydroxy decanoic acid (5-HD), a mitochondrial membrane ATP-sensitive potassium channel blocker (NARI+5-HD), respectively. The left ventricular pressure, lactate dehydrogenates (LDH) in coronary effluent, superoxide dismutase (SOD) and malondialdehyde (MDA) in myocardium, and myocardial infarct area were measured. Nari above $2.5 \mu \mathrm{mol} / \mathrm{L}$ improved the recovery of left ventricular function, decreased LDH in coronary effluent, and reduced myocardial infarct area. The SOD activity was increased and MDA was decreased in Nari-treated myocardium. The cardioprotective effect of Nari was canceled by GLI and 5-HD. In conclusion, Nari has a cardioprotective effect against I/R injury, which may be carried out through activating ATP-sensitive potassium channels in both cell and mitochondrial membrane, and enhancing myocardial antioxidant capacity.
\end{abstract}

Keywords Naringenin; Cardiac protection; Ischemia/reperfusion; ATP-sensitive potassium channels; Antioxidation 


\section{Introduction}

Myocardial ischemia is a common clinical issue. Myocardial damage and cell necrosis will occur after persistent ischemia. Early reperfusion therapy can reduce the injury of myocardial ischemia, but also induce reperfusion injury, such as the decrease of ventricular systolic function, the decrease of myocardial antioxidant capacity, the increase of infarct size, and arrhythmia (Schulze et al. 2003).

Naringenin (Nari) is a kind of flavanone deriving from dry outer layer of immature or nearly mature Rutaceae grapefruit. A great number of researches have proved that Nari has broad spectrum of biological effects, such as hypolipidemic, anti-oxidation, anti-bacterial, anti-inflammation, anti-tumor, and anti-atherosclerosis. It was reported that Nari has protective effect on diabetes, staphylococcus pneumonia and ischemic cranial nerves injury, which might related to the antioxidation, anti-apoptosis and anti-inflammation of Nari (Dou et al. 2013; Kannappan and Anuradha 2010; Lou et al. 2012; Rahigude et al. 2012; Tsai et al. 2012; Yoshida et al. 2013; Zhang et al. 2013). Recently, one study showed that Nari exerts anti-ischemic effects on rat heart which is likely to be mediated by the activation of mitochondrial BK channels (Testai et al. 2013). And another study reported that Nari attenuated pressure overload-induced cardiac hypertrophy (Zhang et al. 2015). However, the mechanism of Nari protection on heart against I/R injury is far from clear.

ATP-sensitive potassium channels located in the sarcolemma ( $\left.\operatorname{sarcoK}_{\mathrm{ATP}}\right)$ and mitochondria $\left(\right.$ mitoK $\left._{\mathrm{ATP}}\right)$ of cardiac myocytes are important regulators of cardiac function and play crucial roles in cardiac protection of ischemia pre-conditioning (IPC). Potassium channel openers can mimic the cardio- protection and the $\mathrm{K}_{\mathrm{ATP}}$ channel blocker can abolish the effect of IPC (Andersen et al. 2012; Powers et al. 2014). However, the link between Nari and $K_{\text {ATP }}$ channel in myocardial $I / R$ injury 
remains unclear. Therefore, it is intriguing to determine whether $\mathrm{K}_{\mathrm{ATP}}$ channel is involved in the cardioprotection of Nari. The aim of the present study was to investigate the protective effect of Nari on heart against $I / R$ injury and the role of $\mathrm{K}_{\mathrm{ATP}}$ channel in Nari effect by using Langendorff technique in rat.

\section{Materials and methods}

\section{Animal and reagents}

Fifty-four adult male Sprague-Dawley rats weighing 280 320 g were provided by the Experimental Animal Center of Hebei Province, China. All animal experiments were conducted in compliance with the Guide for the Care and Use of Laboratory Animals (National Research Council, 1996).

Nari, glibenclamide (GLI), 5-hydroxydecanoate (5-HD) were purchased from Sigma, USA. LDH kit, TTC reagents, superoxide dismutase (SOD) and and malondialdehyde (MDA) kits were purchased from Nanjing Jiancheng Bioengineering Institute, China.

\section{Langendorff and cardiac function recording}

Rats were anesthetized with sodium pentobarbital (50 mg/kg, ip), and the hearts were quickly excised and mounted on a Langendorff apparatus via the aorta for retrograde perfusion with Krebs-Henseleit $(\mathrm{K}-\mathrm{H})$ solution at constant pressure (10 $\mathrm{kPa}$ ). The K-H solution (in mmol/L) was composed of: $\mathrm{NaCl} 118.3, \mathrm{KCl} 4.7, \mathrm{CaCl}_{2}$ 1.8, $\mathrm{MgSO}_{4}, 7 \mathrm{H}_{2} \mathrm{O} 1.2, \mathrm{KH}_{2} \mathrm{PO}_{4} 1.2, \mathrm{NaHCO}_{3} 25$, Glucose 11.1. The solution was continuously gassed with $95 \% \mathrm{O}_{2}$ and $5 \% \mathrm{CO}_{2}(\mathrm{pH} 7.4)$, and maintained at $37^{\circ} \mathrm{C}$. A water-filled latex balloon connected to a pressure transducer (Gould P23Db) was introduced into the left ventricle through the atria to record isovolumic left ventricular 
pressure. The balloon volume was adjusted to achieve a stable left ventricular end-diastolic pressure (LVEDP) of 3-10 $\mathrm{mmHg}$ during initial equilibration. Left ventricular developed pressure (LVDP), LVEDP, maximum rates of rise and decline of developed left ventricular pressure ( $\mathrm{LVdP} / \mathrm{dt}_{\max }$ and $\left.-\mathrm{LVdP} / \mathrm{dt}_{\max }\right)$, heart rate $(\mathrm{HR})$ and coronary flow (CF) were monitored with the PowerLab system (AD Instruments Ltd., Australia).

\section{Animal group and experimental protocols}

Rats were randomly divided into four groups: (1) control group (CON): after stabilization for $20 \mathrm{~min}$ with $\mathrm{K}-\mathrm{H}$ solution, the hearts were subjected to $30 \mathrm{~min}$ no-flow global ischemia followed by 60 min of reperfusion (or 120 min reperfusion for the determination of infarct size); (2) Naringenin group (NARI): the hearts were pretreated with $1.25,2.5,5,10,20$, or $40 \mu \mathrm{mol} / \mathrm{L}$ Nari 5 min before ischemia and reperfusion, respectively; (3) Nari+glibenclamide group (NARI+GLI): the hearts were given $10 \mu \mathrm{mol} / \mathrm{L}$ glibenclamide, a ATP-sensitive potassium channel $\left(\mathrm{K}_{\mathrm{ATP}}\right)$ antagonist, before $10 \mu \mathrm{mol} / \mathrm{L}$ Nari pretreatment; (4) Nari+5-hydroxydecanoate group (NARI+5-HD): the hearts were given $100 \mu \mathrm{mol} / \mathrm{L}$ 5-HD, a selective mitochondria $\mathrm{K}_{\mathrm{ATP}}$ antagonist, before $10 \mu \mathrm{mol} / \mathrm{L}$ Nari pretreatment.

\section{Measurement of coronary effluent lactate dehydrogenase}

During the experiment, coronary effluent was collected at $5 \mathrm{~min}$ before ischemia, $10 \mathrm{~min}, 20 \mathrm{~min}, 30 \mathrm{~min}$ and $60 \mathrm{~min}$ during reperfusion, respectively. The content of lactate dehydrogenase (LDH) was measured with a spectrophotometer in accordance with instruction of the lactate dehydrogenase kit. 


\section{Measurement of myocardial superoxide dismutase and malondialdehyde}

At the end of the Langendorff experiment, left and right ventricles were separated. Fifty milligrams of myocardium was quickly removed from the apex of the heart and was homogenized with normal saline $\left(0{ }^{\circ} \mathrm{C}\right)$ in a glass homogenizer. Twenty percentages of homogenates were centrifuged for $10 \mathrm{~min}$ at $4000 \mathrm{rpm}$ and supernatants were collected. Protein content of the myocardium was measured by the coomassie blue protein assay (NanJing jiancheng Scientific and Technical Company, China), and the activity of SOD and content of MDA in myocardium were measured by the xanthine oxidase method and penthiobarbital acid method (NanJing jiancheng Scientific and Technical Company, China), respectively.

\section{Measurement of infarct area}

At the end of the Langendorff experiment, the heart was quickly removed and the ventricles were cut into 5 slices along the atrioventricular groove from the base to apex of heart. The slices were placed in $10 \%(\mathrm{wt} / \mathrm{vol})$ TTC solution and incubated for $15 \mathrm{~min}$ at $37^{\circ} \mathrm{C}$ in the dark. Then the reaction was quenched with distilled water and the slices were fixed for 2 hours in formalin. The pictures were taken with a digital camera and analyzed with a computer running image analysis system (MoticMed 6.0 Xiamen). The infarct area was expressed as the percentage of infarct area in ventricular area.

\section{Statistical analysis}

All data were expressed as mean $\pm \mathrm{SD}$. Analysis of variances (ANOVA) followed by a Dunnett's post hoc test were used to compare the data among multiple groups. 
The paired $t$ test was used for data within the same group. The $P<0.05$ was considered significant.

\section{Results}

\section{Protective effect of Nari on I/R rat heart}

Effect of Nari on cardiac function There were no significant differences of functional parameters of heart between $\mathrm{CON}$ and NARI groups under basic non-ischemic conditions $(P>0.05)$. During $\mathrm{I} / \mathrm{R}$, the LVDP, $\mathrm{LVdP} / \mathrm{dt}_{\max }, \quad-\mathrm{LVdP} / \mathrm{dt}_{\max }$ and CF were decreased, while LVEDP was increased significantly, but the change of the parameters was smaller in NARI group $(10 \mu \mathrm{mol} / \mathrm{L})$ than those in CON group. The recovery of cardiac function from I/R was improved in NARI group compared to CON group $(P<0.05-0.01$, Fig. 1$)$.

After 60 min reperfusion, $\mathrm{LVDP}, \mathrm{LVdP} / \mathrm{dt}_{\max },-\mathrm{LVdP} / \mathrm{dt}_{\max }$ and $\mathrm{CF}$ were higher, while LVEDP was lower at 2.5, 5, 10, 20 and $40 \mu \mathrm{mol} / \mathrm{L}$ Nari in NARI group than those in CON group $(P<0.05-0.01$, Fig.2).

Effect of Nari on LDH in coronary effluent After I/R, LDH in coronary effluent was lower at $2.5,5,10,20$ and $40 \mu \mathrm{mol} / \mathrm{L}$ Nari than that in CON group $(P<0.05-0.01$, Fig.3)

Effect of Nari on infarct size After 120 min reperfusion, myocardial infarct size was lower at $2.5,5,10,20$ and $40 \mu \mathrm{mol} / \mathrm{L}$ Nari than that in CON group $(P<0.05-0.01$, Fig.3)

All results suggest that Nari has a cardiac protection against $I / R$ injury in a 
concentration manner.

\section{Effect of Nari on the SOD activity and MDA content}

After I/R, myocardial SOD activity was higher at 2.5, 5, 10, 20 and $40 \mu \mathrm{mol} / \mathrm{L}$ Nari than that in CON group $(P<0.05-0.01$, Fig.4). MDA content was lower at 2.5, 5, 10, 20 and $40 \mu \mathrm{mol} / \mathrm{L}$ Nari than that in CON group $(P<0.05-0.01$, Fig.4). The results suggest that Nari increases the antioxidation of the hearts.

\section{Influence of GLI and 5-HD on the cardioprotection of Nari}

The enhanced recovery of cardiac function, reduced infarct area, decreased LDH and MDA, and increased SOD by $10 \mu \mathrm{mol} / \mathrm{L}$ Nari were reversed totally by GLI, the non-specific $\mathrm{K}_{\mathrm{ATP}}$ channels antagonist, and 5-HD, the selective mitochondria $\mathrm{K}_{\mathrm{ATP}}$ antagonist $(P<0.05-0.01$, Fig.5, Fig.6, and Fig.7). These data suggest that the cardioprotective effect of Nari $(10 \mu \mathrm{mol} / \mathrm{L})$ is carried out through activation of ATP-sensitive channels in cardiomyocytes.

\section{Discussion}

This study investigated the cardioprotective effect of Nari against I/R injury and the mechanism underlying Nari cardioprotection in rats. The results showed that Nari has a protective effect on rat heart, promoting the recovery of cardiac function from $\mathrm{I} / \mathrm{R}$, increasing coronary blood flow after reperfusion, decreasing LDH in coronary effluent, and reducing myocardial infarct area. In addition, Nari increased myocardial SOD activity and decreased MDA content after $\mathrm{I} / \mathrm{R}$, which means Nari enhances myocardial antioxidation capacity. The cardioprotective effect of Nari was abolished 
by glibenclamide, a non-specific ATP-sensitive potassium channel antagonist, and 5-hydroxydecanoate, a specific mitochondrial membrane ATP-sensitive potassium channel antagonist, suggesting the activation of ATP-sensitive potassium channels in the cell membrane and mitochondrial membrane are related to the cardioprotective effect of Nari.

Oxygen radicals produced during I/R are important factors for myocardial injury (Jeroudi et al. 1994). During myocardial I/R, production of radicals increased through several pathways, such as xanthine oxidase and other oxidative enzymes located in myocardial mitochondria and vascular endothelial cells, neutrophil respiratory burst, and catecholamine oxidation. The excessive radicals will change the fluidity and permeability of myocardial cell membrane and subcellular membranes, and affect the integrity and function of the cells (Ruiz-Gines et al. 2000). It is known that SOD is an important enzyme in antioxidant enzyme system of the body, and SOD activity usually reflects the body's antioxidation activity. The MDA is the final product of the radical chain reaction of lipid peroxidation, and its content reflects the level of oxygen free radicals as well as the strength and speed of lipid oxidation. So, the measurement myocardial SOD and MDA can be used to evaluate the oxidation activity of myocardium (Zweier and Talukder 2006). The present study showed that Nari increased myocardial SOD activity and decreased MDA content during I/R, indicating Nari enhances myocardial antioxidation capacity and myocardial tolerance to $\mathrm{I} / \mathrm{R}$ injury. It is known that both naringin and Nari from citrus fruits are strong antioxidants and have protective effect on the body (Jung et al. 2003; Renugadevi and 
Prabu 2009). It was reported that naringin exerted its protective role via regulating antioxidative capacity by increasing SOD activity (Singh et al. 2004) and by up-regulating the mRNA expression of SOD (Jeon et al. 2001). So we have reason to speculate that Nari might increase SOD capacity through up-regulating the gene expression of SOD. The enhancement of antioxidation capacity might be an important mechanism for Nari cardioprotection against I/R injury.

$\mathrm{K}_{\mathrm{ATP}}$ channel is a class of potassium channels in cell or mitochondrial membranes, which couples with metabolic and electrical activities, and plays an important role in the regulation of cellular function (Papp et al. 2005; Wei et al. 2004). As a weak inward rectifier potassium channel, $\mathrm{K}_{\mathrm{ATP}}$ is inhibited by increasing intracellular ATP and activated by enhancing diphosphate (ADP). The $\mathrm{K}_{\text {ATP }}$ channel is closed under normal physiological conditions, but it opens and allows potassium efflux to hyperpolarize the membrane in the conditions of myocardial ischemia, decreased intracellular ATP concentration, and ischemic metabolite accumulation. During this time, cardiac action potential duration is shortened, voltage-dependent calcium current is diminished, and myocardial contractility is decreased (Papp et al. 2005). From the view point of energy saving, opening $\mathrm{K}_{\mathrm{ATP}}$ attenuates muscle contraction and reduces energy consumption, producing a protective effect on the myocardium. Numerous studies demonstrated that cardiac $\mathrm{K}_{\mathrm{ATP}}$ plays a pivotal role in the cardiac protection induced by ischemia preconditioning or hypoxia preconditioning (Andersen et al. 2012; Papp et al. 2005). There are two kinds of $\mathrm{K}_{\mathrm{ATP}}$ in cardiomyocytes: sarcolemmal $\mathrm{K}_{\mathrm{ATP}}\left(\operatorname{sarcK}_{\mathrm{ATP}}\right)$ and mitochondrial membrane ATP 
(mitoK $\left.{ }_{\mathrm{ATP}}\right)$. Researches found that mitoK $_{\mathrm{ATP}}$ plays a more important role in myocardial ischemic preconditioning (Jin et al. 2012) and executes a variety of functions, such as maintain mitochondrial $\mathrm{K}^{+}$balance, control mitochondrial matrix volume, and keep homeostasis of trans-membrane potential and $\mathrm{pH}$ gradient during energy production by mitochondrial oxidative phosphorylation (Wei et al. 2004). It was reported that 5-HD, an inhibitor of mitochondrial ATP-sensitive potassium channels, abolished the protective effect of chronic intermittent hypobaric hypoxia treatment on heart against $\mathrm{I} / \mathrm{R}$ ( $\mathrm{Bu}$ et al. 2015). In this study, the cardioprotective effects of Nari was canceled by non-specific $\mathrm{K}_{\mathrm{ATP}}$ blocker Gli and specific mitoK $\mathrm{ATP}_{\mathrm{AT}}$ blocker 5-HD, which suggests Nari protects heart against I/R injury through opening of $\mathrm{K}_{\mathrm{ATP}}$, especially mitoK $\mathrm{K}_{\mathrm{ATP}}$.

On the whole, this study demonstrated the cardioprotective effect of Nari against $\mathrm{I} / \mathrm{R}$ injury through activation of $\mathrm{K}_{\mathrm{ATP}}$ channels for the first time. The study provided new data and experimental basis for further study of Nari cardioprotection, which may has potential value for the clinical application of Nari.

\section{Author contribution}

Prof. Yi Zhang and Hui-Jie Ma designed the research and revised the manuscript; Li-Min Meng performed the research, analysed the data and wrote the manuscript; Hui Guo and Qian-Qian Kong helped with part of the research. 


\section{References}

Andersen, A., Povlsen, J.A., Botker, H.E., and Nielsen-Kudsk, J.E. 2012. Ischemic preconditioning reduces right ventricular infarct size through opening of mitochondrial potassium channels. Cardiology, 123(3): 177-180. doi: 10.1159/ 000342481.

Bu, H.M., Yang, C.Y., Wang, M.L., Ma, H.J., Sun, H., and Zhang, Y. 2015. K(ATP) channels and MPTP are involved in the cardioprotection bestowed by chronic intermittent hypobaric hypoxia in the developing rat. J. Physiol. Sci. 65(4): 367-376. doi: 10.1007/s12576-015-0376-5.

Dou, W., Zhang, J., Sun, A., Zhang, E., Ding, L., Mukherjee, S., Wei, X., Chou, G., Wang, Z.T., and Mani, S. 2013. Protective effect of naringenin against experimental colitis via suppression of Toll-like receptor 4/NF-kappaB signalling. Br. J. Nutr. 110(4): 599-608. doi: 10.1017/S0007114512005594.

Jeon, S.M., Bok, S.H., Jang, M.K., Lee, M.K., Nam, K.T., Park, Y.B., Rhee, S.J., and Choi, M.S. 2001. Antioxidative activity of naringin and lovastatin in high cholesterol-fed rabbits. Life Sci. 69(24): 2855-2866.

Jeroudi, M.O., Hartley, C.J., and Bolli, R. 1994. Myocardial reperfusion injury: role of oxygen radicals and potential therapy with antioxidants. Am. J. Cardiol. 73(6): 2B-7B.

Jin, C., Wu, J., Watanabe, M., Okada, T., and Iesaki, T. 2012. Mitochondrial K+ channels are involved in ischemic postconditioning in rat hearts. J. Physiol. Sci. 62(4): 325-332. doi: 10.1007/s12576-012-0206-y. 
Jung, U.J., Kim, H.J., Lee, J.S., Lee, M.K., Kim, H.O., Park, E.J., Kim, H.K., Jeong, T.S., and Choi, M.S. 2003. Naringin supplementation lowers plasma lipids and enhances erythrocyte antioxidant enzyme activities in hypercholesterolemic subjects. Clin. Nutr. 22(6): 561-568.

Kannappan, S., and Anuradha, C.V. 2010. Naringenin enhances insulin-stimulated tyrosine phosphorylation and improves the cellular actions of insulin in a dietary model of metabolic syndrome. Eur. J. Nutr. 49(2): 101-109. doi: 10.1007/ s00394-009-0054-6.

Lou, C., Zhang, F., Yang, M., Zhao, J., Zeng, W., Fang, X., Zhang, Y., Zhang, C., and Liang, W. 2012. Naringenin decreases invasiveness and metastasis by inhibiting TGF-beta-induced epithelial to mesenchymal transition in pancreatic cancer cells. PLoS One, 7(12): e50956. doi: 10.1371/journal.pone.0050956.

Papp, Z., Csapo, K., Pollesello, P., Haikala, H., and Edes, I. 2005. Pharmacological mechanisms contributing to the clinical efficacy of levosimendan. Cardiovasc. Drug Rev. 23(1): 71-98.

Powers, S.K., Smuder, A.J., Kavazis, A.N., and Quindry, J.C. 2014. Mechanisms of exercise-induced cardioprotection. Physiology (Bethesda), 29(1): 27-38. doi: 10.1152/physiol.00030.2013.

Rahigude, A., Bhutada, P., Kaulaskar, S., Aswar, M., and Otari, K. 2012. Participation of antioxidant and cholinergic system in protective effect of naringenin against type-2 diabetes-induced memory dysfunction in rats. Neuroscience, 226: 62-72. doi: 10.1016/j.neuroscience.2012.09.026. 
Renugadevi, J., and Prabu, S.M. 2009. Naringenin protects against cadmium-induced oxidative renal dysfunction in rats. Toxicology, 256(1-2): 128-134. doi: 10.1016/ j.tox.2008.11.012.

Ruiz-Gines, J.A., Lopez-Ongil, S., Gonzalez-Rubio, M., Gonzalez-Santiago, L., Rodriguez-Puyol, M., and Rodriguez-Puyol, D. 2000. Reactive oxygen species induce proliferation of bovine aortic endothelial cells. J. Cardiovasc. Pharmacol. 35(1): 109-113.

Schulze, C.J., Wang, W., Suarez-Pinzon, W.L., Sawicka, J., Sawicki, G., and Schulz, R. 2003. Imbalance between tissue inhibitor of metalloproteinase-4 and matrix metalloproteinases during acute myocardial [correction of myoctardial] ischemia-reperfusion injury. Circulation, 107(19): 2487-2492. doi: 10.1161/ 01.CIR.0000065603.09430.58.

Singh, D., Chander, V., and Chopra, K. 2004. Protective effect of naringin, a bioflavonoid on glycerol-induced acute renal failure in rat kidney. Toxicology, 201(1-3): 143-151. doi: 10.1016/j.tox.2004.04.018.

Testai, L., Martelli, A., Marino, A., D'Antongiovanni, V., Ciregia, F., Giusti, L., Lucacchini, A., Chericoni, S., Breschi, M.C., and Calderone, V. 2013. The activation of mitochondrial $\mathrm{BK}$ potassium channels contributes to the protective effects of naringenin against myocardial ischemia/reperfusion injury. Biochem. Pharmacol. 85(11): 1634-1643. doi: 10.1016/j.bcp.2013.03.018.

Tsai, S.J., Huang, C.S., Mong, M.C., Kam, W.Y., Huang, H.Y., and Yin, M.C. 2012. Anti-inflammatory and antifibrotic effects of naringenin in diabetic mice. J. Agric. 
Food Chem. 60(1): 514-521. doi: 10.1021/jf203259h.

Wei, K., Min, S., and Long, C. 2004. Cardioprotective effects of mitochondrial KATP channels activated at different time. Chin. Med. J. (Engl), 117(5): 647-651.

Yoshida, H., Watanabe, W., Oomagari, H., Tsuruta, E., Shida, M., and Kurokawa, M. 2013. Citrus flavonoid naringenin inhibits TLR2 expression in adipocytes. J. Nutr. Biochem. 24(7): 1276-1284. doi: 10.1016/j.jnutbio.2012.10.003.

Zhang, N., Yang, Z., Yuan, Y., Li, F., Liu, Y., Ma, Z., Liao, H., Bian, Z., Zhang, Y., Zhou, H., Deng, W., Zhou, M., and Tang, Q. 2015. Naringenin attenuates pressure overload-induced cardiac hypertrophy. Exp. Ther. Med. 10(6): 2206-2212. doi: 10.3892/etm.2015.2816.

Zhang, Y., Wang, J.F., Dong, J., Wei, J.Y., Wang, Y.N., Dai, X.H., Wang, X., Luo, M.J., Tan, W., Deng, X.M., and Niu, X.D. 2013. Inhibition of alpha-toxin production by subinhibitory concentrations of naringenin controls Staphylococcus aureus pneumonia. Fitoterapia, 86: 92-99. doi: 10.1016/j.fitote.2013.02.001.

Zweier, J.L., and Talukder, M.A. 2006. The role of oxidants and free radicals in reperfusion injury. Cardiovasc. Res. 70(2): 181-190. doi: 10.1016/j. cardiores. 2006.02.025.

\section{Figure Legends}

Fig. 1. Effect of Naringenin $(10 \mu \mathrm{mol} / \mathrm{L})$ on functional parameters in isolated rat hearts subjected to 30-min global ischemia and 60-min reperfusion. BL: baseline; 
$\mathrm{CON}$ : control group; NARI: NARI group; Data were expressed as mean $\pm \mathrm{SD}, n=$ 6 for each group, $* P<0.05 * * p<0.01$ vs $\mathrm{CON}$.

Fig 2. Effect of different concentration of Nari on cardiac function of rat at 60-min reperfusion after 30-min global ischemia. 0: Control group; Data were expressed as mean $\pm \mathrm{SD}, n=6$ for each group, ${ }^{*} p<0.05 * * p<0.01 v s 0$, \# $p<0.05 \# \# p<0.01$ vs 2.5 $\mu \mathrm{mol} / \mathrm{L}$.

Fig. 3. Effect of different concentration of Nari on LDH (A) and infarct area (B) in isolated rat hearts subjected to 30-min global ischemia followed by 60-min reperfusion. 0: control group; Data were expressed as mean $\pm \mathrm{SD}, n=6$ for each group, ${ }^{*} p<0.05 * * p<0.01$ vs 0 , \#\# $p<0.01 v s 2.5 \mu \mathrm{mol} / \mathrm{L},++p<0.01 v s 5 \mu \mathrm{mol} / \mathrm{L}$.

Fig. 4. Effect of different concentration of Nari on SOD activity (A) and MDA content (B) in myocardium after 30-min global ischemia followed by 60-min reperfusion. 0: control group; Data were expressed as mean $\pm \mathrm{SD}, n=6$ for each group, $* p<0.05 * * p<0.01 v s 0$.

Fig.5 Influence of GLI and 5-HD on the cardiac protection of Naringenin $(10 \mu \mathrm{mol} / \mathrm{L})$ in isolated rat hearts after 30-min global ischemia and 60-min reperfusion. CON: control group; NARI: NARI group; NARI-GLI: NARI-GLI group; NARI-5HD: NARI-5HD group; Data were expressed as mean $\pm \mathrm{SD}, n=6$ for each group, ${ }^{* *} p<0.01$ vs CON, \#\#p<0.01 vs NARI.

Fig.6. Influence of GLI and 5-HD on the Infarct area of Naringenin $(10 \mu \mathrm{mol} / \mathrm{L})$ in isolated rat hearts after 30-min global ischemia and 60-min reperfusion. CON : control group; NARI : NARI group; NARI-GLI: NARI-GLI group; NARI-5HD: NARI-5HD group; Data were expressed as mean $\pm \mathrm{SD}, n=6$ for each group, ${ }^{* *} p<0.01$ vs CON, \#\# $p<0.01$ vs NARI. 
Fig.7. Influence of GLI and 5-HD on the LDH (A), SOD (B) and MDA (C) action of Naringenin $(10 \mu \mathrm{mol} / \mathrm{L})$ in isolated rat hearts after 30-min global ischemia and 60-min reperfusion. CON: control group; NARI: NARI group; NARI-GLI: NARI-GLI group; NARI-5HD: NARI-5HD group; Data were expressed as mean $\pm \mathrm{SD}, n=6$ for each group, ${ }^{*} p<0.05,{ }^{* *} p<0.01$ vs CON, \# $p<0.05, \# \# p<0.01$ vs NARI. 

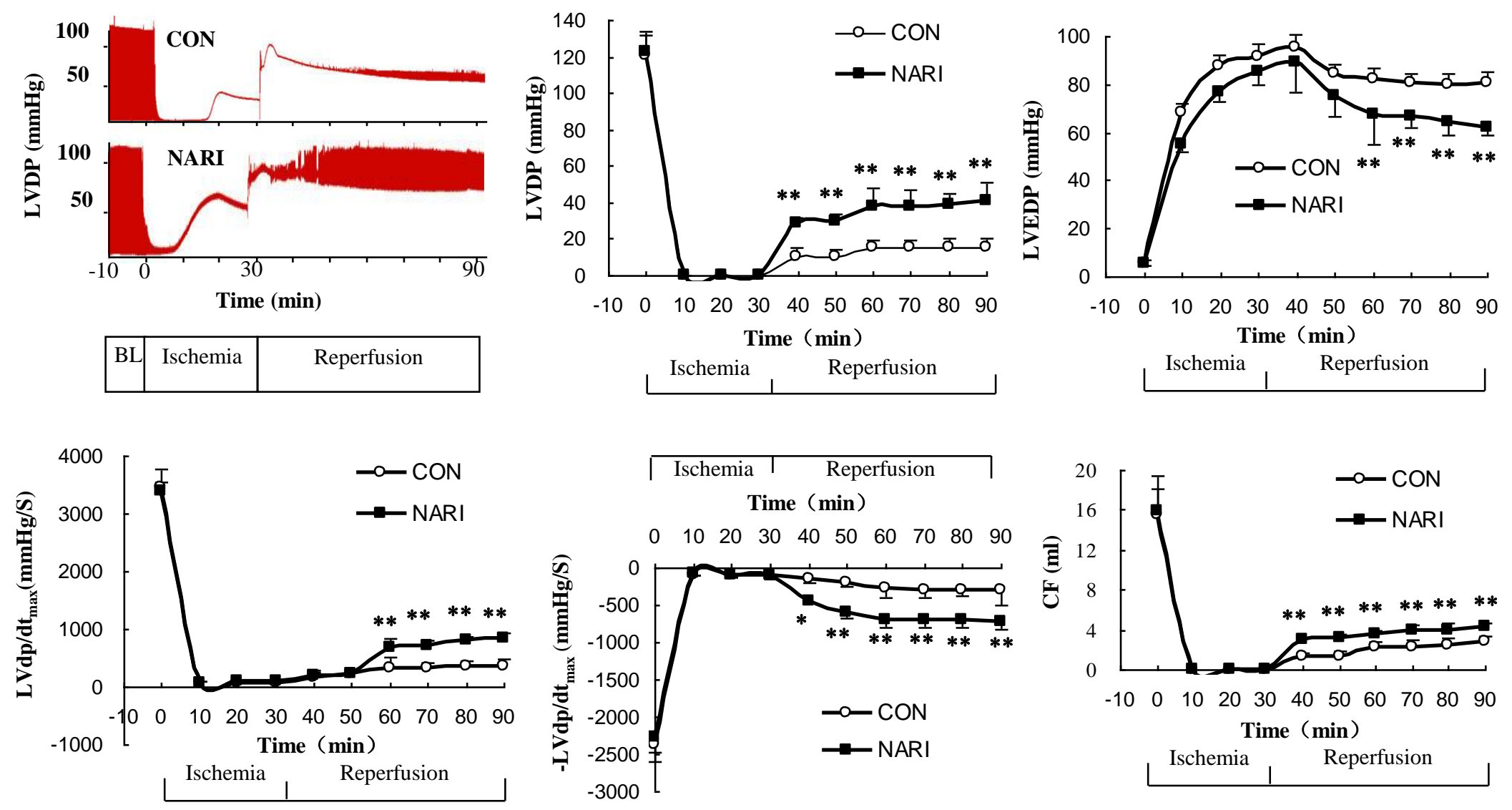

Figure 1 

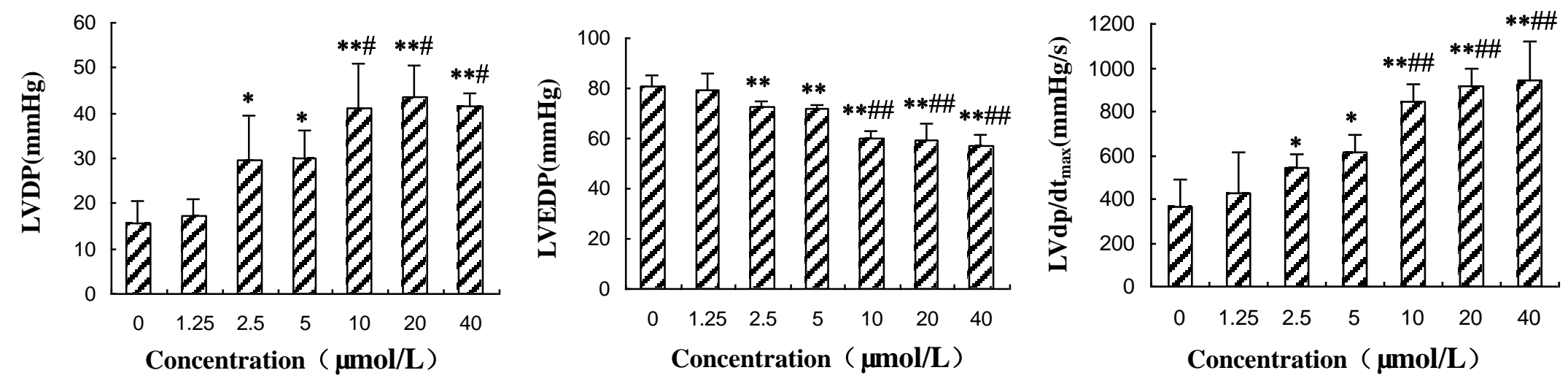

Concentration ( $\mu \mathrm{mol} / \mathrm{L})$
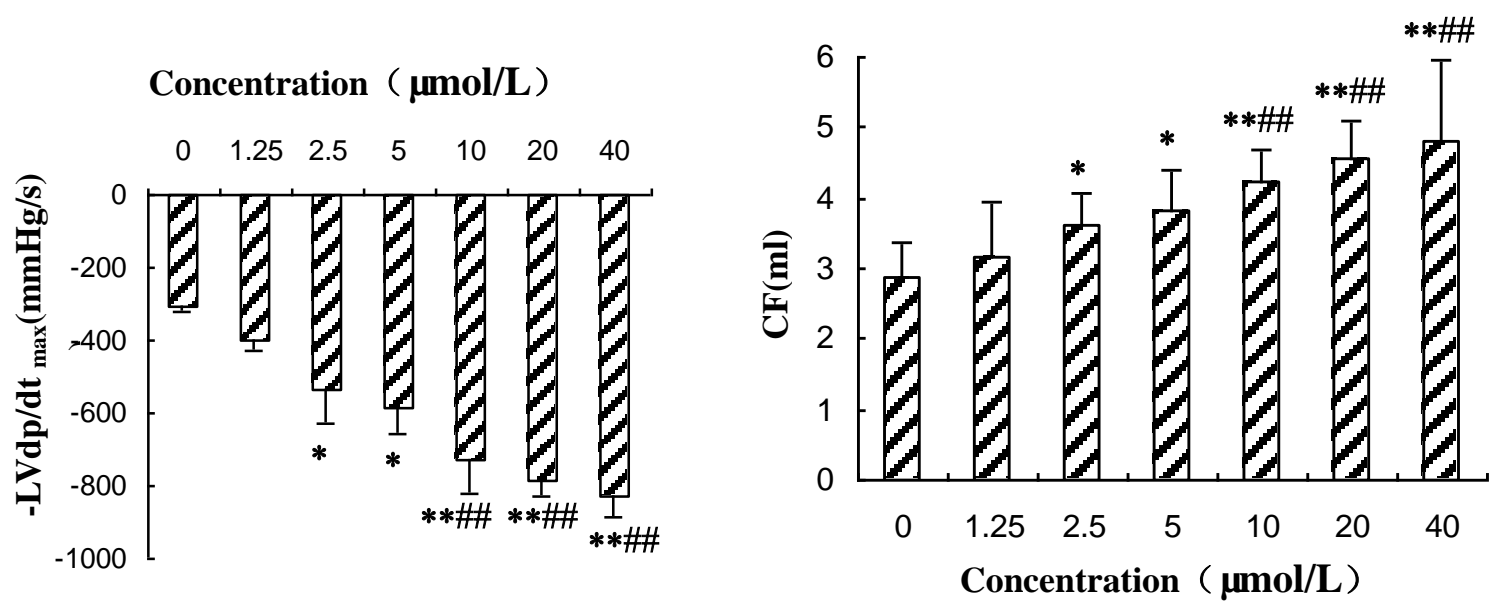

Figure 2 
A

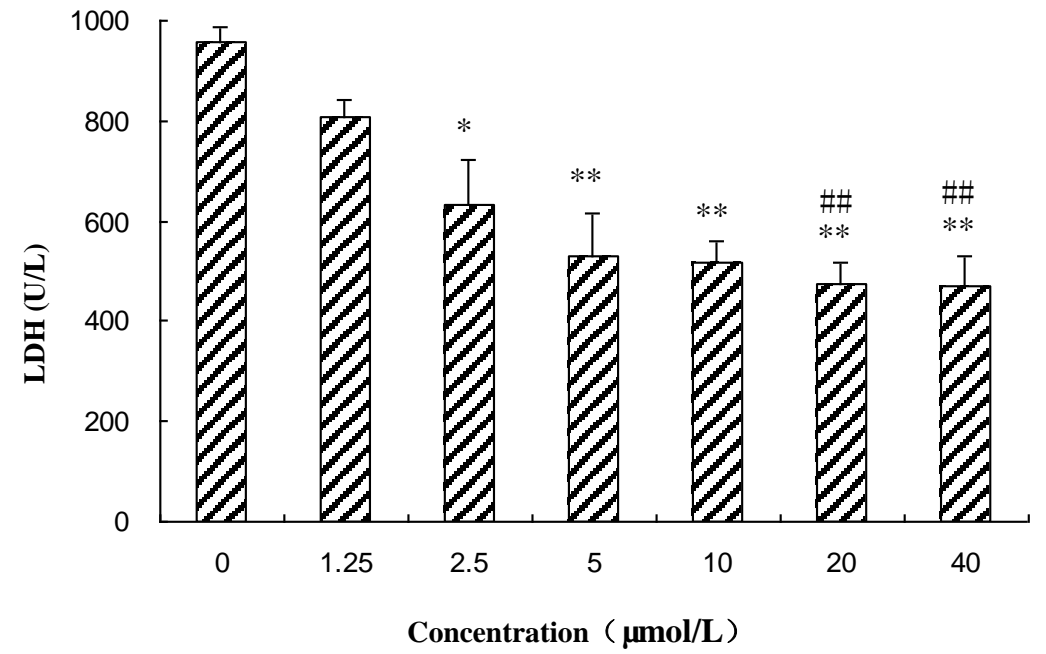

B

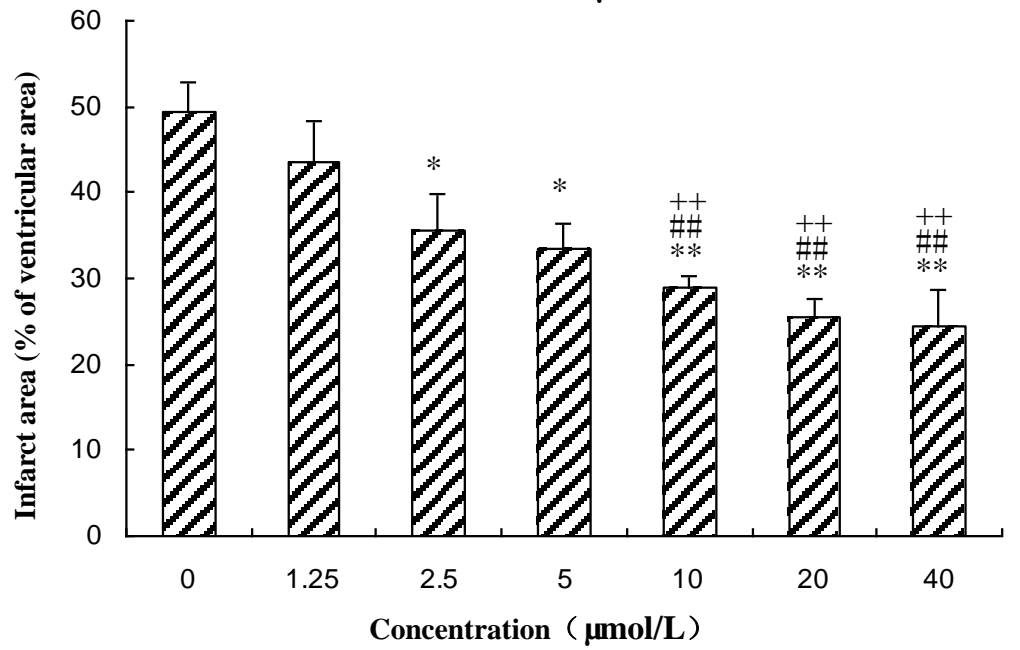

https://mc06.manuscriptcentral.com/cjpp-pubs 

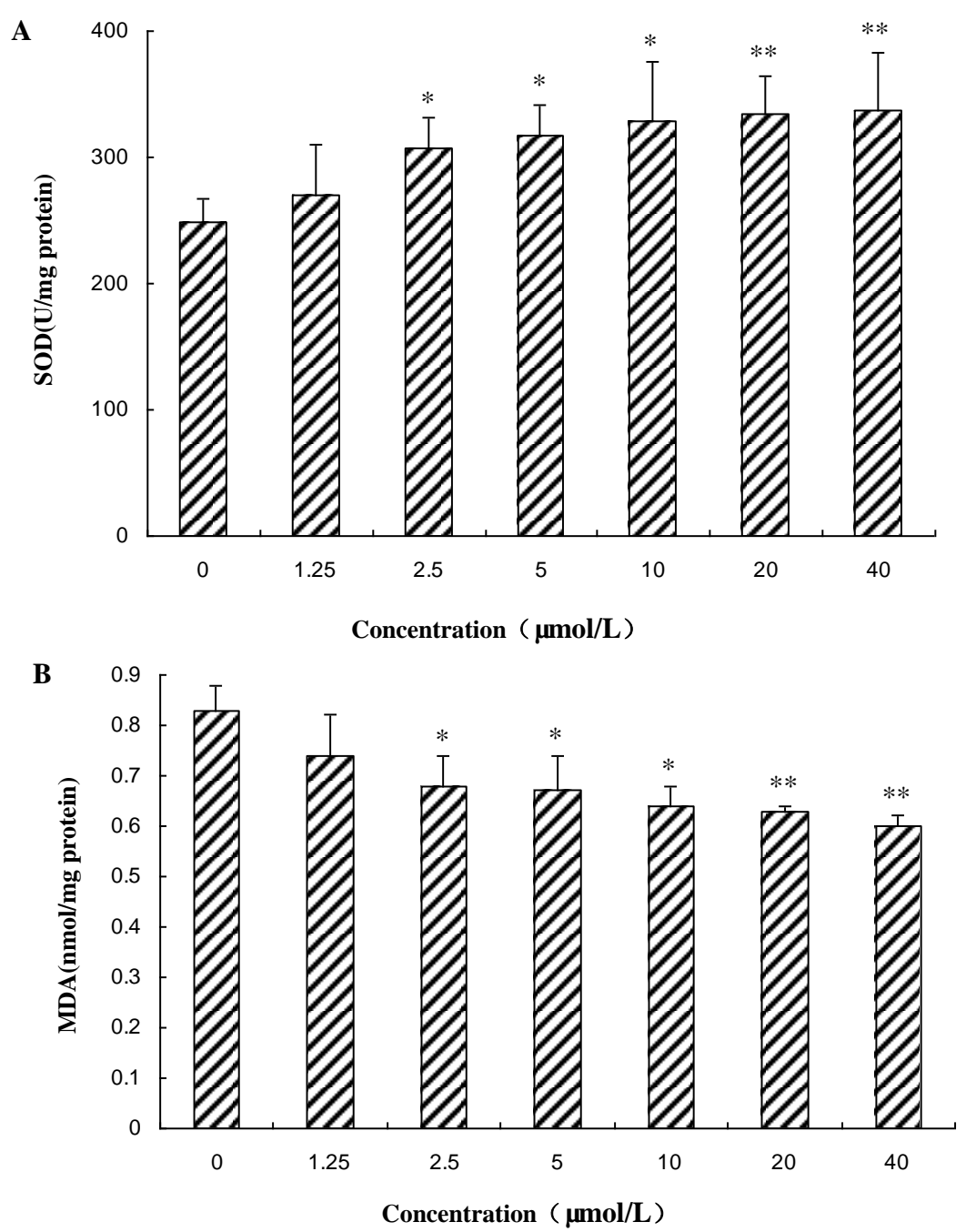

Figure 4

https://mc06.manuscriptcentral.com/cjpp-pubs 

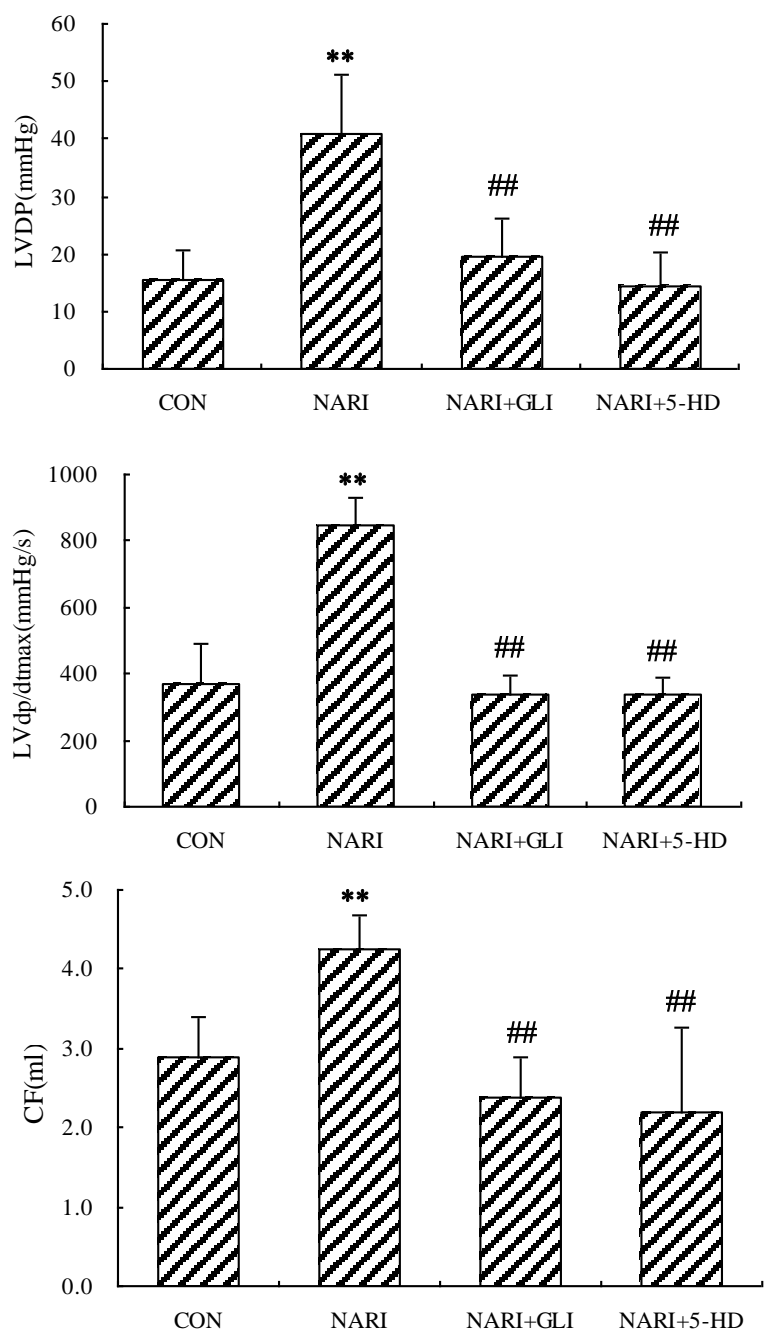

Figure 5

https://mc06.manuscriptcentral.com/cjpp-pubs 


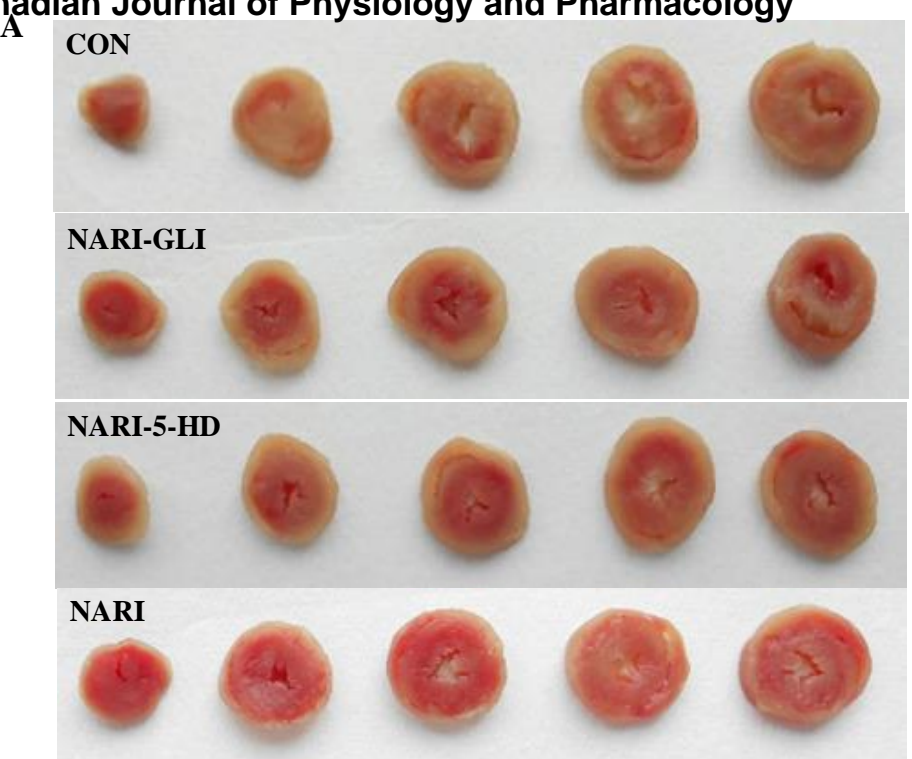

B

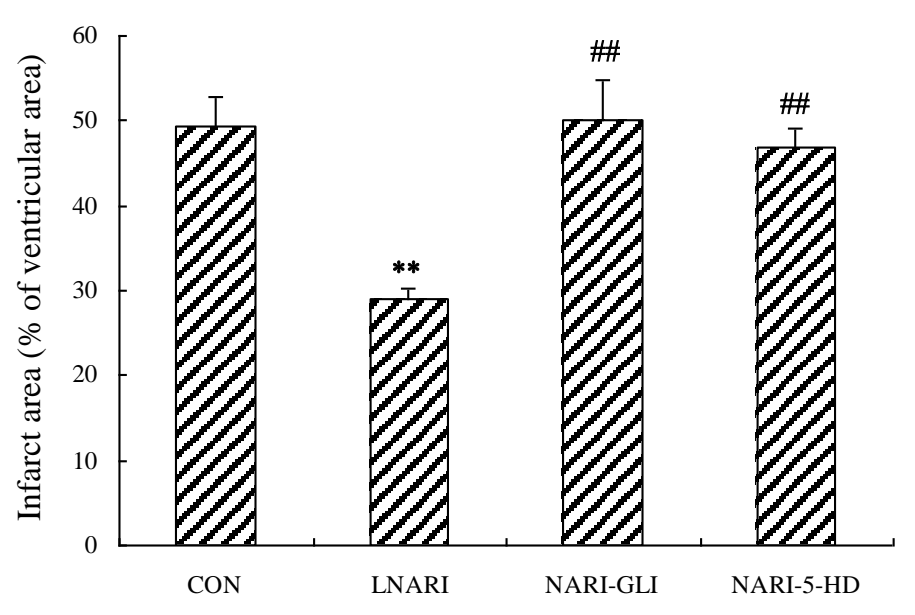

Figure 6

https://mc06.manuscriptcentral.com/cjpp-pubs 
A

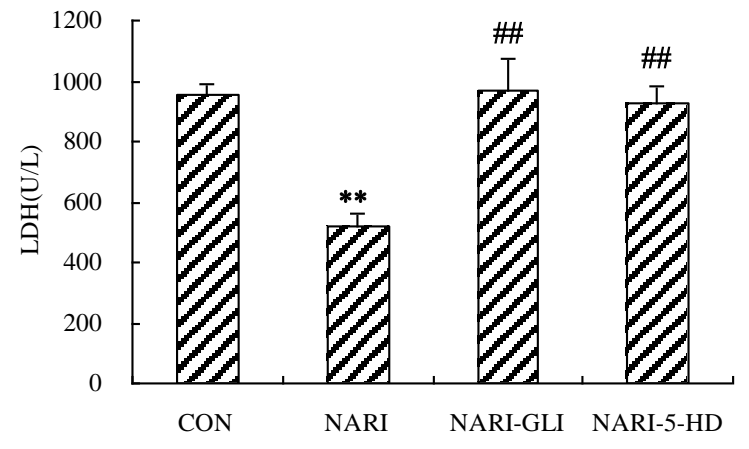

B

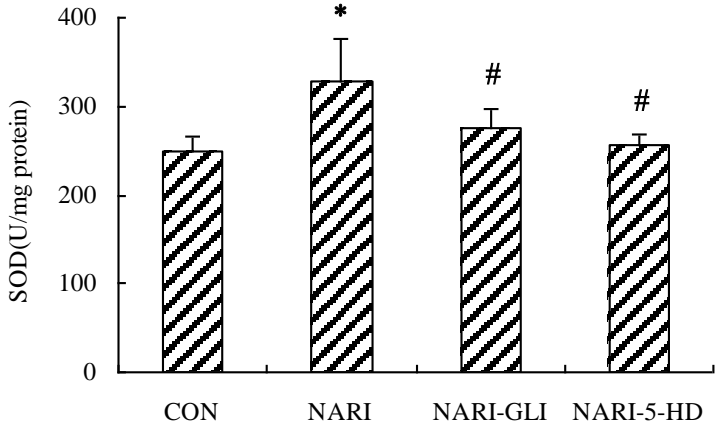

C

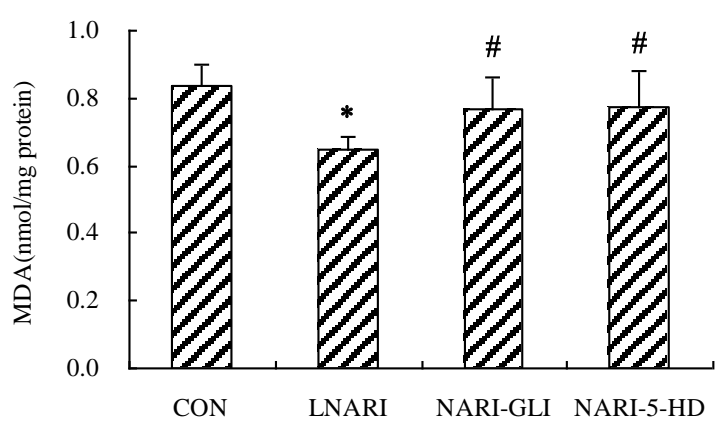

Figure 7 